\title{
EL SISTEMA FAMILIAR Y EL TRABAJO SOCIAL
}

\author{
MARIA JOSE ESCARTIN CAPARROS
}

Profesora de Trabajo Social EUTS de Alicante

\section{INTRODUCCION}

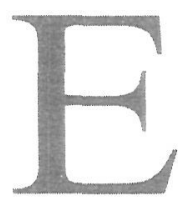

1 Trabajo Social Familiar, fundamental en nuestra profesión, constituye la forma más antigua de TS. Nació de las instituciones públicas y privadas que a través del tiempo han ayudado a las familias en sus problemas. Pero es a partir de Mary Richmond cuando se destaca como fundamental la importancia de la familia en cualquier forma de actuación social. De hecho, es la «Family Service Association of America» la creadora de la primera Escuela de TS.

A lo largo de la historia del TS, diversos autores han abordado la intervención en la unidad familiar, incluso, desde las perspectivas más dispares (recuérdese la importancia de la línea sistémica en el diagnóstico y comprensión de las dificultades familiares, desde el punto de vista relacional, hasta el punto que una de las más importantes Escuelas en Terapia Familiar, la de Palo Alto - California- en la que destaca el nombre de Virginia Satir, hace años que viene desarrollando como especialidad esta modalidad del TS).

Desde la perspectiva psicosocial, tienen gran importancia las escuelas británicas.

Podemos tomar como definición operacional la que propone Bradbock de que la familia es «una suma de individuos situados en ciertas relaciones descriptivas (es decir, observables) entre sí». Pero no podemos obviar el hecho de que la familia es, además, como grupo social primario, con unas funciones precisas en la sociedad, intra y extrafamiliares, de una importancia fundamental en los procesos de cambio o desarrollo (o incluso de involución) de una comunidad.

El TS familiar implica la actuación en un sistema natural, la familia en su totalidad, con el objetivo de modificar la problemática de todos o algunos de sus miembros, pero trabajando con todo el grupo familiar, sus necesidades y recursos internos, aunque sin aislarla del entorno social del que forma parte y del que tendremos que utilizar algunos de sus recursos para ayudar a la familia (o sistema multicliente). 


\section{LA FAMILIA COMO SISTEMA}

Aproximarnos al estudio de la familia, desde una perspectiva sistémica, supone esbozar, aunque sea someramente, algunos de sus rasgos más característicos.

La familia es una complejidad organizada en un «holón» compuesto de subsistemas en mutua interacción ${ }^{1}$. Ackerman la define como una unidad básica de salud y enfermedad.

En cuanto «holón» de personas en interacción, el sistema familiar es más que la suma de sus partes individuales; por tanto, la familia como sistema está vitalmente afectada por cada unidad del sistema, de manera que lo que ocurre a un miembro, de inmediato tiene sus repercusiones en todos los demás y viceversa.

A su vez, las familias son subsistemas de unidades más vastas: la familia extensa, el vecindario, la sociedad como un todo. La interacción con estos «holones» más vastos engendra buena parte de los problemas y tareas de la familia, así como de sus sistemas de apoyo.

Como todos los sistemas y suprasistemas en relación recíproca, la familia y su espacio vital deben ser ecológicos: cada uno tiene sus necesidades y cada uno impacta al otro. Esto ha de equilibrarse si ambos han de seguir siendo funcionales.

La familia y el suprasistema permanecen en equilibrio ecológico mediante un intercambio simbiótico de inputs y outputs, o para decirlo en lenguaje sociológico, a través del equilibrio de funciones ( y los recursos necesarios para cumplir aquellas funciones) que la familia proporciona a la sociedad y de las funciones que la sociedad proporciona a la familia. Es decir, las funciones del sistema familiar deben satisfacer las necesidades de la familia y de sus miembros y también algunas de las necesidades de la sociedad para que la familia sea realmente estructural.

\subsection{Características de la familia como sistema}

1) Totalidad, puesto que es una combinación de partes que se relacionan entre si en constante interacción. Pero hay que observarla en su totalidad y no aisladamente, como formando una red de interacciones, con toda su complejidad. Por ejemplo, como el juego del ajedrez.

2) Circularidad, esto es, en la teoría de sistemas, a los comportamientos no se les puede describir en relación causa-efecto $\mathrm{A}=\mathrm{B}$, sino en relación circular $\mathrm{A}<---->\mathrm{B}$.

${ }^{1}$ Steven Preister, La teoría de sistemas como marco de referencia para el estudio de la Familia, Rev. de Trabajo Soc. de la Un. Cat. de Chile, junio de 1985. 


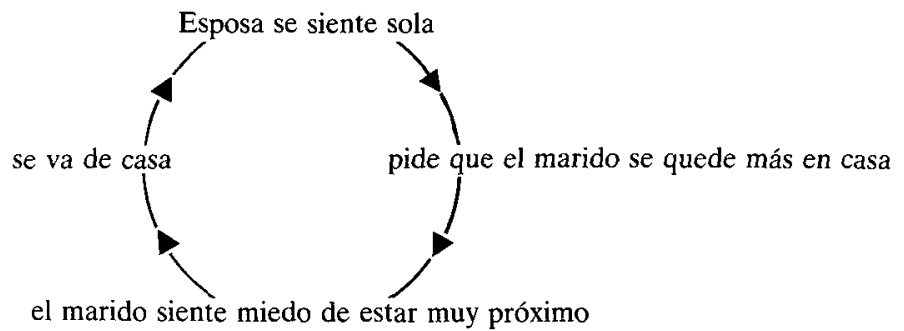

Es decir, cada forma concreta de comportamiento está afectando a las respuestas de los otros y cada comportamiento es contemplado encajando con los otros.

3) Capacidad autocorrectiva u homeóstasis. Como todo sistema, en la familia existe la tendencia a mantener la estabilidad, a la ausencia de cambio, en un sentido morfostático; tiene que ver con los límites.

4) Capacidad de transformación, esto es, una característica que se refiere a la morfogénesis y tiene que ver con el tiempo.

e) Algunos autores, para resolver esa aparente contradicción han aportado la teoría del equilibrio dinámico. Un típico dilema de la familia es estabilidad vs. cambio.

\subsubsection{Estructura de la familia}

Un sistema debe tener una estructura tanto para sobrevivir, como para cumplir sus metas y funciones. La estructura de la familia es la organización de sus relaciones a través de los subsistemas familiares.

La estructura familiar, por tanto, es el conjunto invisible de demandas funcionales que organizan los modos en que interactúan los miembros de la familia.

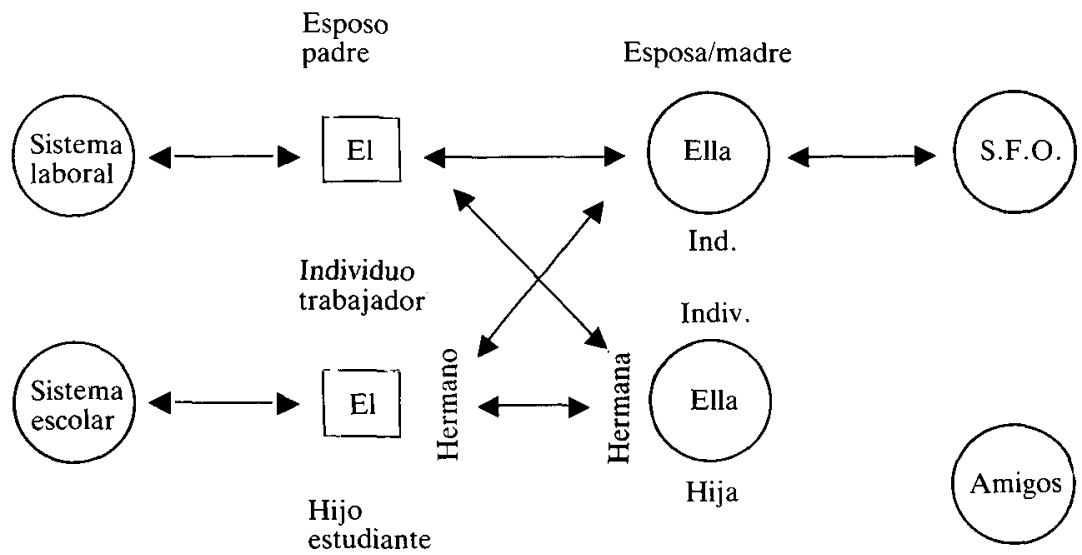


Ciertamente, un individuo es un subsistema de la familia; adicionalmente, otros subsistemas habitualmente identificados son el subsistema marital, el subsistema padre(s)-hijo (s) y el subsistema hermanos. A menudo, los subsistemas familiares son analizados en términos de díadas, el más básico de todos los sistemas sociales. Sin embargo, actualmente, muchos teóricos, particularmente Murray Bowen, consideran el triángulo como la forma estructural más común, aunque disfuncional, de los subsistemas familiares ${ }^{2}$.

Todo esto implica que los límites de los miembros familiares individuales y de los subsistemas familiares son de máxima significación.

\section{NIVELES DE OBSERVACION}
A. Parámetro externo de la familia total
B. Díada marital
C. Subsistema masculino (hombres)
D. Subsistema femenino (mujeres)
E. Tríada padres e hijo
F. Subsistema hijos
G. Sistema de la personalidad individual
H. Límites del ego Intra-psíquico ${ }^{3}$

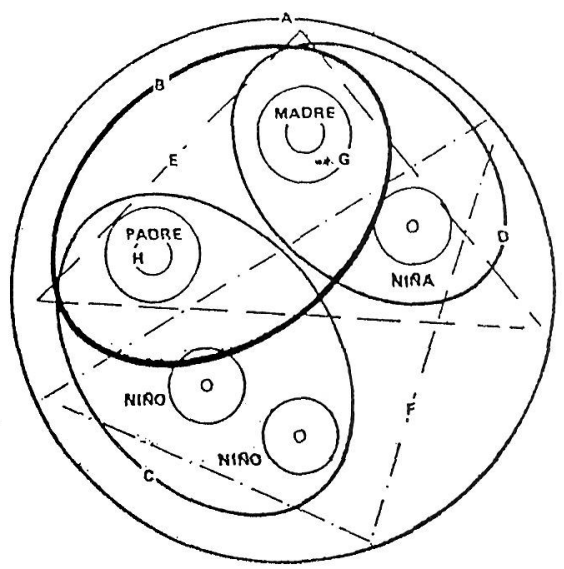

De igual modo que el límite de la familia con su espacio vital, también los límites del subsistema familiar deben ser semipermeables, para permitir una diferenciación e individuación suficiente de los miembros, pero también para permitir un intercambio elegido y apropiado de inputs y outputs. Teóricamente, el grado en el cual las funciones de la familia pueden ser cumplidas adecuadamente, debe correlacionarse directamente con la calidad de los límites de los subsistemas: si los límites son demasiado apretados, el sentido de la familia disminuirá y los miembros de la familia no se ofrecerán entre sí suficiente apoyo; si los límites de los subsistemas son demasiados laxos, los subsistemas no tienen suficiente autonomía para cumplir sus funciones. Así, puede decirse que la intervención con familias puede ser concebida como una intervención en los límites ${ }^{4}$.

2 Murray Bowen, Family Therapy in Clinical Practice, Jason Aronson Ed. Nueva York, 1978.

${ }^{3}$ Steven Preister, op. cit., 1985.

${ }^{4}$ S. Minuchin, Familias y Terapia familiar, Ed. Gedisa, Barcelona, 1977. 


\subsection{Los procesos del sistema familiar: comunicación, roles y reglas}

La estructura de relaciones de la familia es mantenida y se manifiesta a través de los procesos del sistema familiar: la comunicación, los roles y las normas (reglas). El intercambio de inputs y outputs entre los subsistemas familiares es controlado y cumplido a través de estos procesos familiares.

A propósito de la comunicación, nos limitamos aquí a subrayar que empleamos aquí este concepto para denotar el intercambio de símbolos significativos, vocales y gestuales... Puede considerarse que una familia tiene un estilo característico de comunicación. Estos modelos característicos de interacción operan dentro de los límites de la familia y en transacciones con sistemas externos. Por lo tanto, una familia individual tiene un sistema único de modelos de comunicación que influye fuertemente en la conducta de sus miembros.

Los roles son usados continuamente como proceso para ordenar la estructura de relaciones dentro de la familia. Normas o reglas son impuestas para garantizar que se viva a la altura de los roles y se imponen sanciones positivas y negativas para asegurar que las normas se cumplan. Las normas y reglas son la expresión observable de los valores de la familia y/o la sociedad.

Mientras más congruentes son la comunicación, los roles, las reglas y los valores de un sistema familiar, más funcional es éste. Para que exista una familia se requiere una cantidad mínima de congruencia entre estos procesos.

Así, vemos que las funciones, la estructura y procesos familiares están vitalmente interrelacionados.

\subsection{Patrones de comportamiento y sistema de creencias}

Los patrones de comportamiento de una familia definen tanto los límites como la estructura de la familia, siendo aquéllos repetitivos y estables, fruto de las interacciones entre todos los subsistemas familiares y de éstos con el medio. Y es muy importante subrayar que son patrones aprendidos de las familias de origen que los miembros individuales tienden a aportar cuando forman una familia nueva.

Los patrones de comportamiento ofrecen una visión estable de la realidad, proporcionando un sentimiento confortable para los miembros de la familia, así como les ofrece información del interior y del exterior del sistema familiar (transmisión de patrones transgeneracionales).

Como hipótesis podemos decir que los comportamientos problemáticos de las familias forman parte del patrón de comportamiento. Esto surge cuando la familia no se ajusta al cambio. 
Veamos algunos ejemplos de patrones de comportamiento y su relación con los límites.

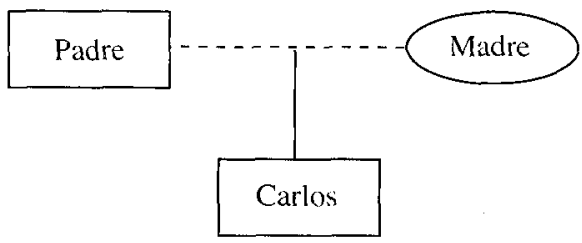

La madre le dice a Carlos que recoja sus juguetes; Carlos se queja al padre y el padre le contesta que haga lo que dice su madre. En este supuesto, hay una frontera clara entre las dos generaciones y los padres se apoyan en sus necesidades y ayudan a crecer a su hijo. P------M

Hijo

En otro supuesto, la madre le dice a Carlos que recoja sus juguetes, Carlos se queja a su padre y el padre le dice a la madre que no sea tan estricta; entonces la madre se retira y Carlos sigue jugando. Este es un patrón de comportamiento sencillo pero con límites imprecisos. P----H

Madre

En este caso, las necesidades de desarrollo de Carlos no se contemplan y los padres entran en conflicto. Carlos puede ser cada vez más rebelde y desafiante. Cuanto más rebelde sea, más peleas de los padres, más conflictos y menos capaces son los padres de poner límites y cuanto menos capaces son de poner límites, más se rebela Carlos y menos se desarrolla.

Esto es un ejemplo de «patrón de relaciones» y en esta situación el comportamiento de Carlos puede ser definido como problema (por la madre, por sus maestros...). Sin embargo, el problema está contenido y es parte del patrón global de comportamiento de la familia.

Existe un procedimiento en la intervención sistemática denominado «connotación positiva» que consiste en reencuadrar las conductas que dentro de la familia están etiquetadas como negativas. Se intenta despolarizar «el bueno» y «el malo» dentro de la familia, proponiendo organizaciones alternativas, positivando lo que es visto como negativo (por ejemplo «mi marido es muy frío», cambiarlo por «es sereno, objetivo...»).

Los sistemas de creencias consisten en una combinación de suposiciones, actitudes, expectativas y valores, aportados por cada padre, transmitidos por sus familias de origen; en ocasiones también pueden producirse por acontecimientos traumáticos en la vida de las familias. En el primer caso, de las creencias que cada uno aporte a la nueva familia va a depender la formación de reglas rígidas, laxas, etc. 
A veces es muy costoso, en la intervención con familias, desafiar los patrones de comportamiento porque es como desafiar también su sistema de creencias.

Veamos dos ejemplos de patrones de conducta y su relación con el sistema de creencias.

Pareja con un niño de dos años muy irascible, con rabietas frecuentes. El abuelo de la familia es un militar rígido que piensa que las mujeres están subordinadas a los hombres. La madre puede pensar que el comportamiento del niño es un reproche por su fracaso al no educarle bien. $\mathrm{O}$, el mismo supuesto, pero el abuelo murió internado en un psiquiátrico con crisis violentas; los padres pueden pensar que el niño ha heredado la locura del abuelo.

\subsection{Cómo ayuda la perspectiva sistémica}

- Ayuda a la familia a contemplar más posibilidades en sus interrelaciones.

- Ayuda a aumentar su repertorio de soluciones.

- Ayuda a los miembros de la familia a que puedan darse cuenta de las paradojas de la familia. Por ejemplo, creencias no respaldadas por comportamientos.

\section{FAMILIA SANA Y FAMILIAS-PROBLEMA: FUNCIONES Y DISFUNCIONES DE LA FAMILIA}

De forma general, puede decirse que una familia «sana», es la que como grupo social primario, cumple unas características:

- Control: modo de mantener el orden o imponer una disciplina que ayude a conseguir las metas establecidas. Se puede lograr de forma democrática o autoritaria. En cualquier caso, cada familia, establece sus reglas del juego para el control del grupo. Hay que destacar la importancia del proceso de toma de decisiones, en el aprendizaje social del niño. El control social, también opera sobre los miembros adultos del grupo.

- Roles: cada miembro de la familia tiene un papel en el grupo familiar, pudiendo ser compartido por diferentes miembros, según las circunstancias.

- Conflicto: como en todo proceso grupal, el conflicto puede surgir por diferentes actitudes en cuanto a tradiciones, valores y reglas introyectados por cada miembro de la pareja, de sus familias de origen, intereses, objetivos, coherencia en la comunicación, etc. Es muy importante para los hijos, la forma en que la familia resuelve y/o resisten los conflictos. 
- Desarrollo personal: cada miembro de la familia necesita que ésta le proporcione las posibilidades para desarrollarse individualmente, de modo que pueda, a su vez, después, formar su propia familia. Las experiencias vividas en las familias de origen, sirven al individuo como pautas para la formación de nuevas unidades familiares.

La familia, como grupo, tiene como fin primordial la socialización de sus miembros, además de la cobertura de las necesidades básicas de todo orden (afectivas, materiales). Cuando un miembro de la familia tiene dificultades personales o sociales, toda la familia se ve implicada. De ahí la importancia del trabajo familiar, de tal forma que no se trata sólo de eliminar las dificultades del individuo, sino de corregir, restaurar y reforzar las capacidades familiares para modificar las dificultades de sus miembros.

Podemos determinar que la familia cumple unas funciones básicas, tanto de cara a los individuos que las componen - funciones intrínsecas-, como hacia la sociedad - funciones extrínsecas-.

Pero como hemos dicho anteriormente, la familia no sólo cumple unas funciones básicas en relación a sus propios miembros, sino que como grupo social que interactúa con otros grupos de su comunidad, tiene una proyección social por lo que debe adoptar formas de comportamiento social basados en los principios de solidaridad, de participación, cooperación y ayuda mutua. Por eso en Trabajo Social, cuando trabajamos en zonas con características semejantes socioeconómicas, culturales y étnicas, suele utilizarse el trabajo con familias para apoyar el trabajo de intervención en la comunidad (lo que Marchioni, por ejemplo llama, como una fase de la intervención comunitaria, la fase de la audición y los coloquios familiares, o, como la forma de intervenir, que está cobrando gran importancia últimamente, a través de las redes sociales - vecinos, asociaciones de padres, etc.).

FUNCIONES HACIA EL INDIVIDUO O INTRINSECAS

\begin{tabular}{|c|c|c|}
\hline Funciones & Disfunciones & Problemas \\
\hline $\begin{array}{l}\text { Vinculación íntima y } \\
\text { permanente entre los } \\
\text { miembros del grupo fa- } \\
\text { miliar. }\end{array}$ & $\begin{array}{l}\text { Evasión de roles pa- } \\
\text { rentales }\end{array}$ & $\begin{array}{l}\text { - Convivencia inesta- } \\
\text { ble } \\
\text { - Relaciones ocasiona- } \\
\text { les } \\
\text { - Poligamia }\end{array}$ \\
\hline & $\begin{array}{l}\text { - Inmadurez e inesta- } \\
\text { bilidad emocional y } \\
\text { afectiva }\end{array}$ & $\begin{array}{l}\text { - Precipitación en la } \\
\text { elección de pareja } \\
\text { - Matrimonios o unio- } \\
\text { nes precoces }\end{array}$ \\
\hline
\end{tabular}




\begin{tabular}{|c|c|c|}
\hline Funciones & Disfunciones & Problemas \\
\hline & & $\begin{array}{l}\text { - Incomunicación de } \\
\text { pareja y/o con los hi- } \\
\text { jos } \\
\text { - Malos tratos de he- } \\
\text { cho y de palabra } \\
\text { - Celos patológicos }\end{array}$ \\
\hline & $\begin{array}{l}\text { - Ausencia de modelos } \\
\text { de roles parentales } \\
\text { por fallecimiento } \\
\text {-separación-aban- } \\
\text { dono o irresponsabili- } \\
\text { dad. }\end{array}$ & $\begin{array}{l}\text { - Personalidades inse- } \\
\text { guras } \\
\text { - Desadaptación in- } \\
\text { fanti] } \\
\text { - Alcoholismo-Droga- } \\
\text { dicción } \\
\text { - Prostitución. Neuro- } \\
\text { sis }\end{array}$ \\
\hline
\end{tabular}

Proporcionar a los hijos las nociones diferenciadas de la sexualidad
Ausencia o distorsión del rol educador de los padres frente a la sexualidad

- Conductas, criterios y aptitudes sexuales basadas en prejuicios culturales y/o frustraciones personales

- Desinterés por ejercer el rol

- Falta de preparación de los padres

- Sobreprotección

$\begin{array}{lll}\begin{array}{l}\text { Regulación y control del } \\ \text { impulso sexual }\end{array} & \text { Anormalidad en la } & - \text { Relaciones sexuales } \\ \text { relación sexual } & \text { indiscriminadas. } \\ & - \text { Prácticas de incesto } \\ & - \text { Violaciones } \\ & - \text { Relaciones sexuales } \\ & \text { precoces } \\ & - \text { Desviaciones sexua- } \\ & \text { les }\end{array}$

Crianza y manutención - Alteración o ausenbiológica y psicológica de los hijos cia de satisfacción de necesidades básicas (biológicas-psicológicas y sociales)
- Desnutrición

- Alimentación inadecuada $y / o$ insuficiente

- Falta de cuidados y prevención de enfermedades

- Carencia o insuficiencia de ingresos económicos

- Hacinamiento

- Promiscuidad

- Vivienda insalubre

- Falta de identificación con el grupo familiar 


\begin{tabular}{cc}
\hline Funciones & Pisfunciones \\
\hline & - Neutralidad afectiva, \\
& y/o rechazo a los hi- \\
& jos \\
& Castigos y malos tra- \\
& tos exagerados \\
& - Pensamiento mágico \\
& e ignorancia \\
\hline
\end{tabular}

Socialización de los hi- - Carencia o transmi- - Modelos negativos jos según pautas cultura- sión distorsionada de les de la familia y la so- modelos en relación ciedad modelos en relación de comportamiento

- Ausencia de pautas culturales

- Ignorancia

- Imposición de patrones rígidos

- Inadaptación a los cambios

\begin{tabular}{|c|c|c|}
\hline & $\begin{array}{l}\text { - Falta de integración } \\
\text { a la familia y a la so- } \\
\text { ciedad }\end{array}$ & $\begin{array}{l}\text { - Prejuicios } \\
\text { - Individualismo } \\
\text { - Posturas doctrinarias } \\
\text { dogmáticas } \\
\text { - Carencia de espíritu } \\
\text { grupal y comunitario }\end{array}$ \\
\hline $\begin{array}{l}\text { Asignación a los hijos de } \\
\text { valores }\end{array}$ & $\begin{array}{l}\text { - Desajuste entre va- } \\
\text { lores dominantes y } \\
\text { valores asignados }\end{array}$ & $\begin{array}{l}\text { - Conflicto de valores } \\
\text { - Resentimiento social } \\
\text { - Marginación social- } \\
\text { individual y grupal } \\
\text { - Distorsión en las as- } \\
\text { piraciones sociales y } \\
\text { ocupacionales (ocu- } \\
\text { paciones irreales) }\end{array}$ \\
\hline $\begin{array}{l}\text { Producción y consumo } \\
\text { como mini unidad eco- } \\
\text { nómica }\end{array}$ & $\begin{array}{l}\text { - Limitación o margi- } \\
\text { nación del proceso } \\
\text { económico }\end{array}$ & $\begin{array}{l}\text { - Dependencia econó- } \\
\text { mica } \\
\text { - Ingresos insuficien- } \\
\text { tes } \\
\text { - Desempleo } \\
\text { - Falta de especializa- } \\
\text { ción } \\
\text { - Agentes sociales im- } \\
\text { productivos }\end{array}$ \\
\hline Control social & $\begin{array}{l}\text { - Alteraciones del } \\
\text { control social }\end{array}$ & $\begin{array}{l}\text { - Falta de conciencia } \\
\text { de la importancia del } \\
\text { control social } \\
\text { - Castigos exagerados } \\
\text { - Ausencia de sancio- } \\
\text { nes } \\
\text { - Sobreprotección fa- } \\
\text { miliar }\end{array}$ \\
\hline
\end{tabular}




Funciones Disfunciones Problemas

$\begin{array}{lcc}\text { Preparación de los hijos } & \text { - Incapacidad o indife- } & \text { Falta de conciencia } \\ \text { para la formación de sus } & \text { rencia para preparar } & \text { de la obligación de } \\ \text { propias unidades fami- } & \text { a los hijos } & \text { preparar a los hijos } \\ \text { liares } & - \text { Vivencias negativas } \\ & \text { en el seno de la fami- } \\ & \text { lia } \\ & \text { Transmisión de pa- } \\ & \text { trones culturales ina- } \\ \text { decuados } & \text { Imposiciones en la } \\ & \text { elección de pareja }\end{array}$

\section{FUNCIONES HACIA LA SOCIEDAD O EXTRINSECAS}

\begin{tabular}{|c|c|c|}
\hline Funciones & Disfunciones & Problemas \\
\hline \multicolumn{3}{|c|}{$\begin{array}{l}\text { Reproducción de los } \\
\text { miembros del grupo fa- } \\
\text { miliar }\end{array}$} \\
\hline & $\begin{array}{l}\text { - Paternidad irrespon- } \\
\text { sable }\end{array}$ & $\begin{array}{l}\text { - Reproducción indis- } \\
\text { criminada (hijos de } \\
\text { diferentes progenito- } \\
\text { res, numerosos hijos } \\
\text { incompatibles con } \\
\text { las condiciones so- } \\
\text { cio-económicas, ges- } \\
\text { tación de hijos con } \\
\text { diferentes mujeres) } \\
\text { - Reproducción de } \\
\text { agentes sociales } \\
\text { anormales } \\
\text { - Infanticidios } \\
\text { - Niños en situación } \\
\text { social irregular (va- } \\
\text { gancia, abandono, } \\
\text { delincuencia, droga- } \\
\text { dicción, alcoholis- } \\
\text { mo, mendicidad, } \\
\text { etc.) }\end{array}$ \\
\hline & $\begin{array}{l}\text { - Morbi-Mortalidad } \\
\text { infantil }\end{array}$ & $\begin{array}{l}\text { - Desnutrición infantil } \\
\text { - Enfermedades infec- } \\
\text { to-contagiosas } \\
\text { - Accidentes e intoxi- } \\
\text { caciones, etc. }\end{array}$ \\
\hline
\end{tabular}




\section{Funciones}

Colaboración en el mejoramiento de la calidad de vida propia y ajena
Disfunciones

Problemas
- Irresponsabilidad familiar frente al ecosistema
- Producción de ruidos excesivos

- Contaminación ambiental

- Destrucción de áreas verdes

- Incumplimiento de normas establecidas para preservar el ambiente

- Desaseo personal y vecinal

- Crianza indiscriminada de animales domésticos

\begin{tabular}{|c|c|c|}
\hline $\begin{array}{l}\text { Relación y ayuda a otras } \\
\text { familias de su comuni- } \\
\text { dad }\end{array}$ & $\begin{array}{l}\text { - Carencia de valores } \\
\text { cooperativos y co- } \\
\text { munitarios }\end{array}$ & $\begin{array}{l}\text { - Individualismo } \\
\text { - Desarraigo por mo- } \\
\text { vilidad permanente } \\
\text { - Indiferencia vecinal } \\
\text { - Conflictos entre ve- } \\
\text { cinos } \\
\text { - Diferencias sociales } \\
\text { y culturales entre ve- } \\
\text { cinos }\end{array}$ \\
\hline
\end{tabular}

\begin{tabular}{|c|c|}
\hline \multirow[t]{2}{*}{$\begin{array}{l}\text { Representación de los } \\
\text { hijos ante la sociedad }\end{array}$} & $\begin{array}{l}\text { - Incapacidad de los } \\
\text { padres para asumir } \\
\text { la representación de } \\
\text { los menores }\end{array}$ \\
\hline & $\begin{array}{l}\text { - Ausencia de iniciati- } \\
\text { vas vecinales frente a } \\
\text { los menores en situa- } \\
\text { ción social irregular } \\
\text { de su comunidad }\end{array}$ \\
\hline
\end{tabular}

- Ignorancia

- Indiferencia

- Orfandad total o abandono

- Irresponsabilidad paterna

- Disgregación familiar

- Falta de solidaridad social

- Ignorancia, indiferencia

- Dificultad de acceso a las autoridades

- Ausencia de líderes comunitarios

- Ausencia de organizaciones de base
Participación en el desarrollo global del país a nivel local, regional y nacional
- Desinterés por la creación, manutención o desarrollo de las organizaciones comunitarias
- Falta de espíritu comunitario

- Desinterés por participar

- Desconocimiento del rol del Municipio 


\begin{tabular}{cc}
\hline Funciones & Problemas \\
\hline & - Desconocimiento del \\
& rol de ciudadano \\
& - Desconfianza en los \\
& objetivos o personas \\
& que dirigen los gru- \\
& pos \\
& - Desprestigio de algu- \\
& nas organizaciones \\
& vecinales \\
& - Falta de capacitación \\
& para la participación \\
\hline
\end{tabular}

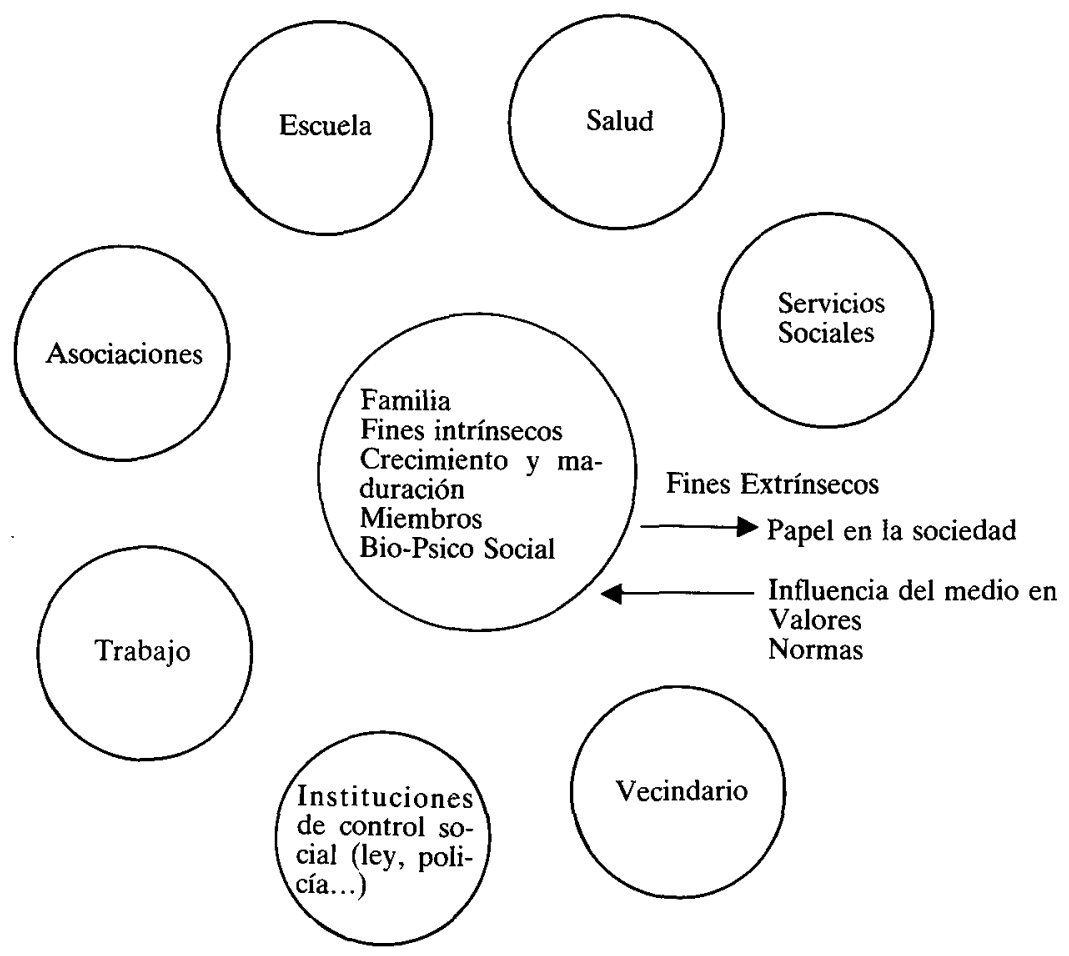

En definitiva, las diferencias entre las familias «sanas» y las familias «sintomáticas» serían:

\section{Familias sanas}

- No tienen en su interior un "chivo expiatorio» (aquel miembro de la familia en el cual el resto proyecta sus problemas, frus- 
traciones, culpas, complejos reprimidos, etc. - por ejemplo «el niño malísimo que dificulta la convivencia familiar»; «el vago del marido que siempre está de copas»...-).

- Se adapta a las presiones externas y al desarrollo somatopsíquico de sus miembros.

- Se acopla de forma madura y adulta.

- Resiste las crisis.

- Generalmente, no necesita recurrir a expertos.

Familias no sanas o sintomáticas

- Tiene en su interior un «chivo expiatorio»

- Es vulnerable a las presiones externas y no se adapta ni posibilita la maduración de sus miembros.

- Presenta dependencia infantil.

- Crisis Cíclicas.

- Necesita ayuda especializada.

\section{APROXIMACION A LA COMPRENSION DE LAS DISFUNCIONES FAMILIARES}

\subsection{Las familias-problema}

Las familias sintomáticas o familias-problema, pueden definirse como aquellas en que la comunicación intrafamiliar se realiza en un lenguaje traducido en síntomas, no hay mensajes verbales, en la medida que esto es lo sano y deseable, sino que por el contrario hay «juego manipulativo", mediante la utilización de emociones provocadas por la presentación de síntomas creados por la familia y mantenidos de forma persistente ${ }^{5}$. A estos conflictos internos debidos a problemas de falta de maduración o ajustes defectuosos suele unirse, en el caso de nuestros clientes, toda una serie de variables incidentes de tipo socioeconómico y cultural: carencia y/o ausencia de recursos económicos, actividades laborales de tipo marginal, deudas, inversión de valores, malas relaciones con el entorno, clientelismo o dependencia de las instituciones oficiales, delincuencia...

Generalmente, este tipo de familias presenta unas características más o menos comunes que, además de las antes mencionadas las distinguen de las familias «sanas». Son las siguientes:

5 J. A. Ríos González, Orientación y Terapia Familiar, Instituto Ciencias del Hombre, Madrid, 1984. 
1. Confusión generacional. En tales familias resulta poco definido el reparto de roles y de conductas de acuerdo con la edad y el momento evolutivo de cada miembro.

Algunos de ellos (o todos) ocupan el lugar que no les corresponde, no hay diferencia de planos, sino un conglomerado donde no hay límites claros.

Este problema toma múltiples formas, desde la ausencia de distancias padres/hijos (por ejemplo, la hermana de 14 años que hace el papel de madre con sus hermanos menores), con una serie de comportamientos iguales y una misma escala de valores: hasta el desempeño por un adolescente de un rol de adulto, ocupando conductas correspondientes a uno de los progenitores. Este tipo de confusión perturba todos los aspectos de la relación familiar impidiendo una correcta y madura asunción de roles por cada uno de los miembros de la familia.

2. Conflictos continuos. La existencia de conflictos que superan un cierto nivel de presencia inevitable en el interior de una familia, constituye un síntoma de cara al exterior, de que en el seno de esa familia se producen anomalías. Los conflictos se refieren tanto a los provocados por las presiones del medio socio-cultural, como por las inadecuadas relaciones entre los miembros, a través de alianzas persistentes y patológicas (triangulaciones manipulativas).

3. Escasa autonomia personal de sus miembros. Se refiere a que la familia impide que los miembros consigan una sana independencia que evite la aparición de hábitos neuróticos como consecuencia de fijaciones infantiles que obstaculizan el crecimiento y la relación de los miembros.

4. Resistencia al cambio. Es una barrera que oculta actitudes hiperprotectoras, dificultades de adaptación, inmadurez personal de los padres, defensas ante las agresiones del medio exterior. Supone una fuente inagotable de mecanismos de racionalización y es uno de los obstáculos más importantes con que tropezamos los trabajadores sociales a la hora de intervenir con este tipo de familias.

Los distintos autores que han estudiado el tema de las familias, han abordado, desde diferentes puntos de vista, las tipologías que pueden existir entre las familias. Aquí vamos a ofrecer algunas de las más interesantes de cara a nuestro trabajo profesional.

- Por el «tipo de encuentro» de los miembros dentro de la familia:

a) Familia distante.

b) Familia simétrica: los miembros están mezclados y confusos. En el seno de la familia existe una dinámica de poder y de sometimiento.

${ }^{6}$ Boll, Family Situations, Univ. Pensylvania Press, Filadelfia, 1976. 
Otra clasificación muy útil para los profesionales del TS es la que hace Boll al estudiar las «situaciones de la Familia» ${ }^{6}$. Es la siguiente:

1. Relaciones afectivas

A. Cariño mal entendido

1. El hogar absorbente o dominante.

2. El hogar demasiado solícito.

3. El hogar indulgente.

-B. Afecto normal

1. El hogar dividido.

C. Discriminación de afectos

1. El hogar con niño favorecido.

2. El hogar imparcial.

D. Inconsistencia en los afectos

1. El hogar de las dependencias.

2. El hogar que no merece confianza.

E. Desplazamiento del afecto

1. El hogar en que aparece un nuevo miembro.

F. Falta de cariño

1. El hogar regañón.

2. El hogar frígido.

3. El hogar negligente.

G. Franca repulsión

1. El hogar con el hijo no deseado.

2. Relación de autoridad entre los miembros:

A. Represión

1. El hogar controlado por la madre.

2. El hogar controlado por el padre.

3. El hogar con demasiadas demandas y exigencias.

B. Anarquía

1. El hogar gobernado por el niño.

C. Confusión

1. El hogar con demasiados amos.

D. Inclinación al equilibrio

1. El hogar democrático.

3. Patrones familiares

A. Volumen

1. La familia numerosa.

2. La familia con un solo hijo. 
B. Organización

1. La familia en que todos cooperan.

2. La familia independiente.

3. La familia incompleta.

C. Actividad

1. La familia nómada.

2. La familia en que todos se apuntan para algo.

3. La familia de intelectuales.

4. La familia que vive colgada.

5. La familia bienhechora de la comunidad.

D. Valores y metas

1. La familia con ambiciones sociales.

2. La familia materialista.

3. La familia muy religiosa.

4. La familia científica.

5. La familia supersticiosa.

6. La familia tradicional.

4. Factores externos

A. Estado socioeconómico

1. Hogar con falta de recursos económicos.

2. Hogar repentinamente rico.

3. Hogar con gran herencia.

4. Familia con características ocupacionales peculiares (actividades marginales, economía sumergida...)

5. Hogar con conflictos de cultura.

6. Hogar con desorganización espaciotemporal.

7. Hogar sostenido por la madre.

8. La familia a la vista del público.

B. Vecindad

1. La familia granjera o campesina.

2. La familia de provincia.

3. La familia de gran ciudad.

4. La familia con lugar de veraneo.

5. La familia con peleas vecinales.

C. Salud

1. El hogar del inválido.

2. El hogar que padece defectos físicos o mentales.

Hay que advertir que, al presentar todas estas tipologías familiares, de lo que se trata es de poder encontrar más indicadores para el diagnóstico de la situación familiar. Como en el caso de los modelos, 
no hay tipos puros de familias y así, en una misma familia, podemos encontrar rasgos de diferentes clasificaciones. Así pues, todas estas clasificaciones no tienen otra función que la de ayudarnos a comprender mejor las características de la familia con la cual trabajamos.

De manera general, los trabajadores sociales siempre intervenimos en familias muy carenciadas tanto en sus procesos internos como externos; familias que habitualmente, no comparten las normas de funcionamiento social, basado en normas de vida familiar. Para algunas familias, acontecimientos tales como el internamiento de un hijo en un centro de protección de menores, las deudas, las separaciones temporales, la falta de vivienda o de trabajo, se han convertido en una forma de vida, de manera que tienen una fuerte resistencia a los cambios que crisis de este tipo podrían suponer nuevas formas de conducta en familias más «funcionales». Algunas familias, incluso han incorporado de tal forma estas crisis cíclicas a su estilo de vida que podría decirse que es una forma de conseguir la continuidad, ya que la familia ha desarrollado pautas de interacción que le permite ajustarse particularmente a su situación y que las diferencia de otros modos de vida de familias «funcionales» o adaptadas según los estándares sociales.

En este sentido, hay muchos trabajadores radicales que cuestionan la manera de que un TS, de clase media, por muy experto que sea en relaciones humanas, pueda ayudar a una familia que está viviendo en la pobreza, en un medio que les ofrece pocas oportunidades de escape a su situación, sea en educación, ocio, actividades culturales u oportunidades de empleo. Estas críticas de la estructura presente del trabajo social apuntan a que muchas de las intervenciones profesionales parecen representar la imposición, por parte de los asistentes sociales, de las normas de vida familiar y social de las clases dominantes sobre los grupos desaventajados.

Además, es un hecho constatado, que en el caso de las familias "clientes forzosos», caracterizadas por ser familias multiproblema o muy marginales y que han tenido experiencias previas negativas con instituciones de Servicios Sociales, los trabajadores sociales tienen muchas dificultades para intervenir ya que encuentran enormes resistencias e incluso hostilidad.

\section{UNA TEORIA DE LA DISFUNCION FAMILIAR: TEORIA DEL «TRIANGULO PERVERSO»}

Teoría del «triángulo perverso». El conocimiento de esta teoría nos ayudará también a reconocer conflictos de relación dentro de las familias.

Los más recientes estudios sobre familias y su tratamiento, pare- 
cen pasar de un análisis diádico de las relaciones de la pareja a un análisis triangular, por considerar que en el contexto familiar existen alianzas o coaliciones en función de la inclusión o exclusión de un tercero.

Esta perspectiva permite captar diferentes etapas dentro de la pareja y abordar desde un punto de vista más social y no exclusivamente psicodinámico, los problemas familiares.

Por triángulo perverso se entiende «una situación de relación en el ámbito de la cual tres miembros de un sistema relacional perteneciente a dos generaciones diversas, se disponen de tal modo que forman una alianza que une a dos de ellos, de generaciones distintas, contra el tercero. Tal alianza se caracteriza por el hecho de ser negada por parte de los dos aliados todas las veces que el tercero se lamenta de ello».

Pueden destacarse unas notas características:

- Las personas que componen el triángulo no son iguales y una de ellas pertenece a una generación diferente de las otras: padre-hijo, superior-inferior, terapeuta-cliente...

- En el proceso interactivo que se establece hay una persona de una generación que forma coalición con la de la otra generación, de tal modo que éstas, aliadas, se sitúan contra la que está en el mismo plano generacional que la primera: madre-hijo contra padre, padre-hija contra madre...

- La coalición establecida se niega cada vez que se plantea de algún modo de forma que «el comportamiento a un cierto nivel que indica la existencia de una coalición, viene cualificado por un comportamiento metacomunicativo que indica la ausencia de tal coalición» ${ }^{7}$.

Este fenómeno de formación de triángulos puede observarse en muchas familias y adoptando formas muy variadas que proyectan una luz clara para la comprensión de conflictos y tensiones.

Si tomamos el triángulo como una unidad de estudio y dividimos una red familiar en sus componentes triangulares, surge una enorme complejidad. En una familia de tamaño medio donde hay dos padres y dos hijos y cada padre tiene a su vez dos padres, este grupo de ocho personas puede componer 56 triángulos. Cualquier persona de la familia se ve comprometida en 21 triángulos familiares simultáneamente. Cada uno de esos 21 triángulos implican una posibilidad de coalición entre dos generaciones. Cuando esas coaliciones se ocultan y se repiten, siguiendo un patrón, estas coaliciones se harán patológicas dando lugar a familias con un alto potencial de perturbación.

Veamos algunas de las situaciones más típicas de triangulación perversa en una familia:

${ }^{7}$ G. Zuk, Terapia familiar y Familias en conflicto, FCE, México, 1988. 
- Triangulación manipulada por los padres en conflicto cuando cada uno de ellos requiere que el hijo se una a él contra el otro progenitor.

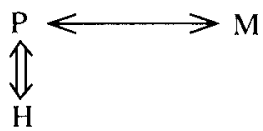

En este caso, cada vez que el hijo se une al que lo reclama, el otro lo define como agresor que lo ataca.<smiles>[M]C1CC1</smiles>

(B)
Esta situación, vista desde el punto de la madre

- Hay un rodeo cuando los padres en conflicto utilizan al hijo sin aliarlo claramente a ninguno de ellos, para mantener la relación de pareja aparentemente con equilibrio y armonía.

En este caso, al hijo se le convierte en un problema que hay que consultar (el hijo síntoma), sin darse cuenta de que ellos mismos refuerzan cualquier aspecto anómalo para poder desviar o tapar sus propios problemas.

Gráficamente se darían estas dos situaciones superpuestas en el tiempo en que la situación $\mathrm{C}$ sirve de tapadera a la situación $\mathrm{D}$, apareciendo muy unidos $\mathrm{P}$ y $\mathrm{M}$ frente al $\mathrm{H}$, siendo la realidad que $\mathrm{P}$ y $\mathrm{M}$ están en conflicto entre sí y el hijo ha sido la víctima del mecanismo de rodeo de sus padres.

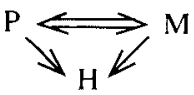

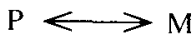

H

- Hay una coalición estable (o triángulo perverso o mortal) cuando se establece una coalición transgeneracional rígida contra el otro progenitor.

\section{Vínculo oculto y negado}

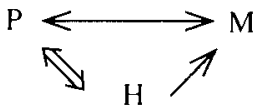

Dentro de estos tres modelos pueden clasificarse las posiciones más frecuentes de la dinámica triangular $\mathrm{P}-\mathrm{M}-\mathrm{H}$, aunque en el caso de más 
hijos, las posibilidades de participar en más de un triángulo perverso complican la realidad y hacen más difícil la tarea de descubrir en qué lugar exacto se halla cada miembro.

En definitiva, incluso teóricos como Bowen, con orientaciones analíticas, afirman que «la estructura básica de cualquier sistema emocional es el triángulo. Cuando la tensión emocional en un sistema de dos personas sobrepasa cierto nivel, forma un triángulo que incluye a una tercera persona, lo que permite que la tensión cambie dentro del triángulo» ${ }^{8}$.

Una concepción de la relación patógena basada triádicamente, debe describir tipos de coaliciones, alianzas o camarillas que tienden a producir "patrones de huida» que, en algún nivel de tensión, producen síntomas de perturbación en los miembros de la familia y en otros grupos.

Una técnica del trabajo con familias basada en la tríada, debe describir como se utiliza el terapeuta como mediador y como toma partido por unos y otros intencionadamente para romper y reemplazar las relaciones conflictivas. En esta técnica, la intervención familiar se define como compuesta por una serie de negociaciones entre el trabajador familiar y la familia en la que ambas partes luchan por obtener el control.

\section{BIBLIOGRAFIA}

S. Minuchin: Técnicas de Terapia familiar, Ed. Paidós, Buenos Aires, 1988.

J. A. Ríos González: Orientación y Terapia familiar, Fund. Inst. Ciencias del Hombre, Madrid, 1984.

M. Walters y P. Papp: La red invisible, Paidós, Buenos Aires, 1991.

S. de Shazer: Pautas de Terapia familiar breve, Paidós, Buenos Aires, 1987.

Watzlawick: Cambio, Herder, Barcelona, 1976. 